> Le développement du pancréas à partir de l'endoderme de l'embryon est un processus complexe incluant la formation des bourgeons pancréatiques puis leur fusion, la différenciation des cellules exocrines et endocrines du pancréas, et la migration de ces dernières pour former les îlots de Langerhans. Ces étapes commencent maintenant à être connues : elles sont sous la dépendance de signaux émis par les tissus adjacents, en particulier par le mésoderme, de l'expression, coordonnée dans le temps et dans des territoires précis, de nombreux facteurs de transcription. Cette dissection de l'ontogenèse du pancréas représente une étape incontournable pour les recherches visant à développer une thérapie cellulaire du diabète. <

\section{Les étapes du développement du pancréas: des pistes pour le traitement du diabète}

Anne Grapin-Botton

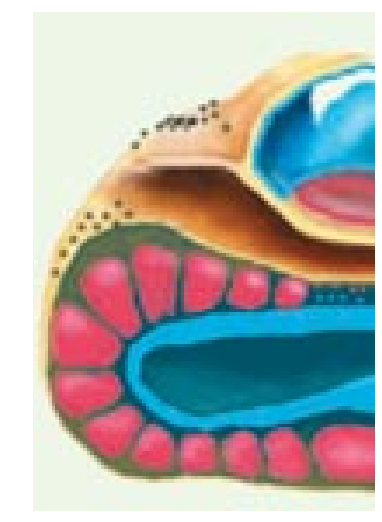

caractérisées dans une minorité de tissus tels que la moelle osseuse, l'épithélium intestinal ou l'épiderme. Leur présence a récemment été mise en évidence dans d'autres organes, notamment dans les muscles et le système nerveux, et il est possible que des cellules de ce type soient représentées dans tous les organes [2]. Des expériences de régénération expérimentale suggèrent qu'il existe des cellules souches dans le pancréas [3] mais on connaît peu leurs caractéristiques et ces cellules n'ont jamais été isolées. Cependant, on sait maintenant que les potentialités de différenciation des cellules souches s'étendent non seulement à l'organe dans lequel elles nichent mais aussi à d'autres organes. Des lignées de cellules souches embryonnaires humaines, qui peuvent être propagées in vitro et qui sont capables de former de nombreux types cellulaires, sinon tous comme leurs homologues murins, ont été développées. Lumelski et al. [4] ont montré que certaines conditions de culture permettaient la différenciation de cellules souches embryonnaires murines en cellules dans lesquelles de l'insuline est présente $(\rightarrow)$. Il s'agit d'une avancée spectaculaire mais ces cellules n'ont pas des taux biologiques d'insuline. La dissection des étapes de l'ontogenèse du pancréas chez l'homme et les animaux modèles ainsi que l'identification des gènes impliqués $\rightarrow) \mathrm{m} / \mathrm{s}$ 2001, $n^{\circ} 10$, p. 1088 nouvelles sources de cellules $\beta$ [2]. Des cellules souches, capables de proliférer et de se différencier pour régénérer les organes chez l'adulte avaient jusqu'alors été bien gement insuffisant pour couvrir la demande [1]. Au cours des années 2000 et 2001 , le renouveau du concept de cellule souche a éveillé l'espoir de disposer à moyen terme de 
devraient permettre de développer des stratégies pour reproduire de plus en plus fidèlement la différenciation des cellules souches, in vitro ou in vivo. La Figure 1 dresse un parallèle entre l'origine embryologique du pancréas et les étapes à récapituler.

\section{Spécification du domaine endodermique du pancréas}

\section{Premiers signes moléculaires}

Bien avant que n'apparaissent les premiers signes morphologiques, alors que l'embryon a une dizaine de somites, certains gènes à homéoboîte s'expriment dans l'endoderme présomptif du pancréas. Un des premiers est le gène $P d x I$ dont l'expression marque initialement les territoires endodermiques qui formeront le pancréas et s'étend progressivement à l'ensemble du duodénum et à l'estomac postérieur $[5,6]$ (Figure 2). Les protéines sécrétées Sonic Hedgehog (Shh) et Indian hedgehog (Ihh) sont exprimées précocement dans la plupart des épithéliums digestifs à l'exception du pancréas (Figure 2). Leur exclusion du domaine pancréatique est importante puisque, lorsqu'on y force l'expression de Shh, le développement de cet organe est anormal [7]. Enfin, des signaux envoyés par les tissus adjacents, en particulier par le mésoderme, sont nécessaires à l'induction des gènes caractéristiques de l'ébauche pancréatique (Figure 3).

\section{Signaux permissifs}

La notochorde, qui est en contact transitoire avec l'endoderme dorsal aux stades précoces de la somitogenèse (Figure 3), envoie des signaux indispensables à l'induc-

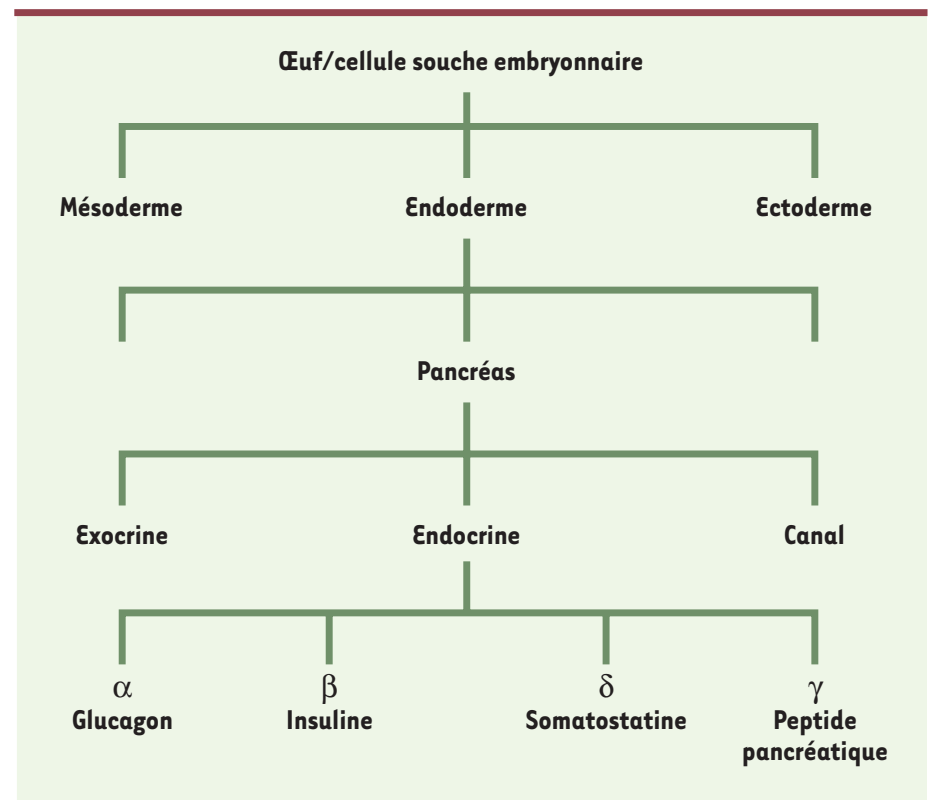

tion de nombreux gènes du pancréas endocrine et exocrine, dont $P d x 1$, et à la répression de l'expression de Shh [8]. L'activine $\beta$ et le FGF2 (fibroblast growth factor), qui miment l'action de la notochorde, pourraient être les intermédiaires biologiques de cet effet [8]. La notochorde s'éloigne ensuite de l'endoderme, poussée dorsalement par l'aorte. L'aorte, mais aussi d'autres vaisseaux sanguins, ont la capacité de stimuler la différenciation des cellules endocrines dans l'endoderme pancréatique [9]. L'association entre glande et vaisseaux sanguins est fonctionnellement importante et cette dépendance précoce est remarquable. Ces signaux sont qualifiés de permissifs car ils sont nécessaires pour le développement du pancréas mais non suffisants pour l'induire dans des domaines antérieurs ou postérieurs au duodénum.

\section{Signaux instructifs}

Des signaux instructifs, capables d'induire les gènes pancréatiques dans d'autres domaines endodermiques, n'ont pas encore été décrits mais des expériences en cours suggèrent que le mésoderme de la splanchnopleure, situé au niveau du futur duodénum, envoie des signaux induisant $P d x I$ dans l'endoderme (Figure 3). Par ailleurs, des signaux qui répriment l'identité pancréatique ont été identifiés. L'endoderme présomptif du foie jouxte celui du pancréas. En présence de signaux du mésoderme cardiaque, nommément des membres de la famille du FGF, l'endoderme de cette région exprime des marqueurs hépatiques alors qu'en son absence, des marqueurs pancréatiques sont induits (Figure 3) [10]. Le mésoderme cardiaque limite donc l'expansion antérieure et ventrale du champ pancréatique.
Figure 1. Récapituler in vitro les étapes de formation du pancréas pour produire en masse des cellules endocrines. Le pancréas est constitué de deux tissus glandulaires étroitement associés, I'un fait de cellules exocrines qui sécrètent des enzymes dans l'intestin et l'autre de cellules endocrines qui sécrètent des hormones dans le sang. Le pancréas exocrine est une glande acineuse alors que le pancréas endocrine est constitué de 4 types cellulaires différents $(\alpha, \beta, \delta, P P)$ regroupés dans les îlots de Langerhans, qui sont répartis dans le mésenchyme entre les branches du pancréas exocrine. Les cellules endocrines et exocrines dérivent toutes de l'endoderme et sont enveloppées de tissu conjonctif et de vaisseaux sanguins, tous deux d'origine mésodermique, et de cellules nerveuses et gliales provenant de la crête neurale. Une cellule souche embryonnaire a la capacité de former des dérivés des trois feuillets embryonnaires. Une approche in vitro de transformation des cellules souches en cellules $\beta$ consisterait à reproduire séquentiellement chaque étape : choix endodermique, activation des facteurs de transcription pancréatiques, différenciation des cellules endocrines. 
De nombreuses malformations congénitales du pancréas - pancréas annulaire, pancreas divisum et hétérotopies pancréatiques - sont déclenchées à ce stade de développement [8]. L'inactivation de Shh et de Ihh (Indian Hedgehog) chez la souris reproduit les trois types de malformations [8] et des mutations du gène $P d x 1$ causent des agénésies et des hypoplasies pancréatiques.

\section{Formation des bourgeons pancréatiques}

Après le début de l'expression des premiers facteurs de transcription caractéristiques du pancréas, trois bourgeons se forment dans le duodénum, l'un dorsal et les deux autres ventro-latéraux (Figure 2). Ces bourgeons fusionneront par la suite pour former un organe unique. Dans certaines espèces, dont la souris et l'homme, I'un des bourgeons ventraux arrête son développement très précocement [9]. Les bourgeons se forment en plusieurs étapes. L'induction est vraisemblablement due à des divisions radiales des cellules de l'épithélium intestinal. II en résulte un épaississement local qui n'est pas présent en l'absence du gène à homéoboîte $H$ lxb-9 [1],
12]. Ce gène est exprimé à la fois dans l'ébauche pancréatique et dans la notochorde. La notochorde étant indispensable à l'expression de nombreux marqueurs pancréatiques (Figure 2), il est difficile de savoir si l'expression de ce gène est requise dans le pancréas ou la notochorde pour le bourgeonnement. Lorsque le gène $P d x l$ est inactivé, les bourgeons pancréatiques se forment mais restent petits et peu ramifiés $[5,6]$. L'expression ectopique de ce gène dans d'autres zones de l'épithélium digestif induit, en revanche, la formation de bourgeons [13]. Ce gène n'est donc pas indispensable à l'induction du bourgeonnement mais semble conférer aux bourgeons la capacité d'envahir le mésenchyme, une activité qui dépend des métalloprotéases et des molécules d'adhérence. Cette capacité s'accompagne d'un renforcement des jonctions entre cellules épithéliales, relayé notamment par une augmentation de l'expression de la $\varepsilon$-cadhérine et sa redistribution vers les surfaces latérales des cellules [14]. Le facteur de transcription à homéoboîte Proxl pourrait aussi jouer un rôle important : ce gène est exprimé transitoirement dans les bourgeons du foie et du pancréas. Il a été montré que, dans le cas du foie, Proxl est nécessaire au bourgeonnement [15].
A

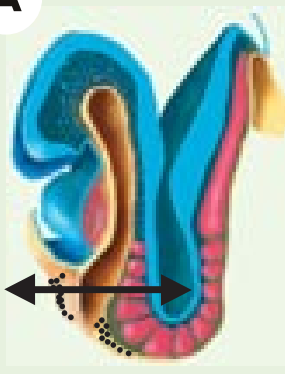

B

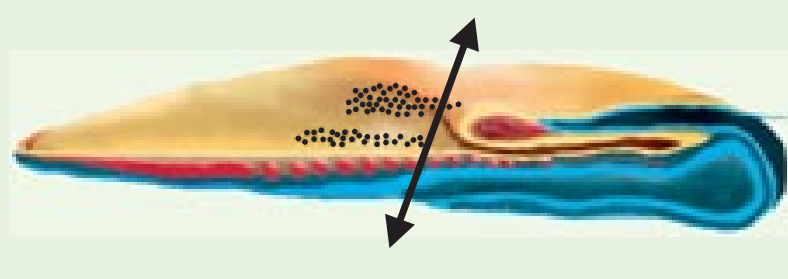

C

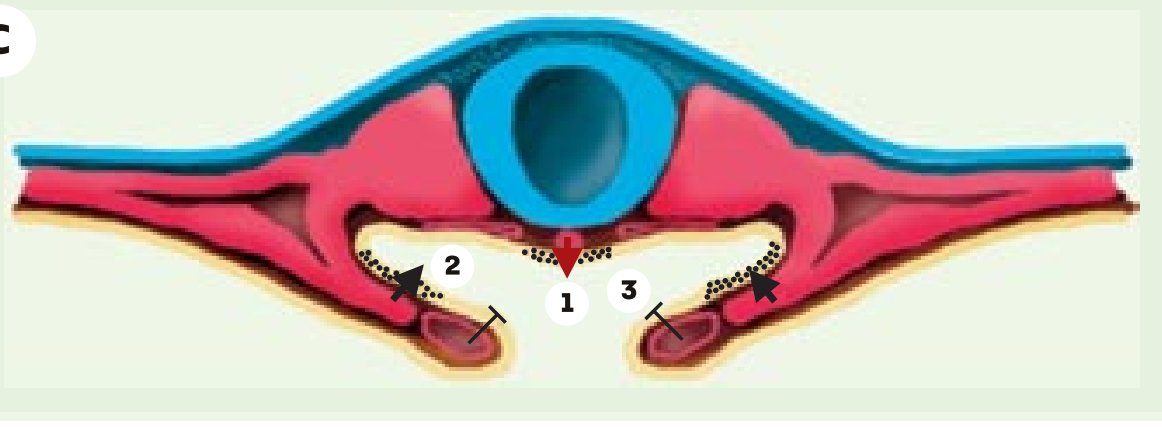

Figure 2. Des signaux des tissus adjacents induisent le développement du pancréas. Les territoires présomptifs des bourgeons du pancréas (grains noirs) sont représentés chez un embryon de souris à $\varepsilon 8,5$ ( $A$ ) et chez un embryon de poulet de 12 somites (B). L'endoderme, le mésoderme et l'ectoderme sont respectivement représentés en jaune, rouge et bleu. C. Une section transversale au niveau de la double flèche montre l'influence des tissus mésodermiques adjacents. La notochorde (1) envoie des signaux permissifs à l'endoderme du futur bourgeon dorsal. Les aortes, latérales à la notochorde à ce stade, fusionneront de façon médiane et prendront le relais. Les bourgeons ventraux reçoivent l'influence instructive du mésoderme splanchnopleural (2) tandis que le mésoderme cardiaque induit le développement hépatique au détriment de celui du pancréas (3).

\section{Différenciation des cellules exocrines et endocrines du pancréas}

\section{Relations de lignage} entre cellules endocrines et exocrines

La différenciation des différents types de cellules commence avant même la formation des bourgeons. Le glucagon, première hormone à être exprimée, est présent dès 9 jours embryonnaires (દ9) chez la souris (Figure 4). L'insuline apparaît quelques heures après, suivie de la somatostatine et du peptide pancréatique à $\varepsilon 15$. Les premiers signes de différenciation exocrine, l'expression du facteur de transcription p48-PTF-1, apparaissent à $\varepsilon 10$ [16]. La proportion entre cellules endocrines et exocrines est réglée par des protéines 
sécrétées. La follistatine, un inhibiteur des membres de la famille du TGF $\beta$ sécrété par le mésenchyme pancréatique, augmente le nombre de cellules exocrines aux dépens des cellules endocrines [17]. Comme dans d'autres organes, le système Notch/Delta est un effecteur membranaire de ce choix dichotomique des cellules de l'endoderme du pancréas. L'inactivation de cette voie de signalisation au niveau du ligand (Delta), du récepteur Notch ou des relais intracellulaires induit la différenciation précoce de cellules endocrines [18, 19]. Les facteurs de transcription à domaine basique hélice-boucle-hélice (bHLH), HES-1, neurogénine 3 ( $\mathrm{Ngn} 3$ ) et NeuroD sont des relais intracellulaires séquentiels de ce signal. Ngn3 et NeuroD promeuvent la différenciation des cellules endocrines aux dépens de celle des cellules exocrines lorsque leur expression est forcée dans l'ensemble du champ [18-20]. Par ailleurs, $\mathrm{Ngn} 3$ active la sortie du cycle de prolifération. L'activation précoce forcée de ce gène dans l'ensemble de l'organe entraîne une déplétion en progéniteurs exocrines et endocrines qui se manifeste par une hypoplasie pancréatique. Le syndrome d'Alagille, qui est causé par des mutations dans le gène Jagged, qui code pour un ligand activant Notch, comprend également des anomalies pancréatiques [21].

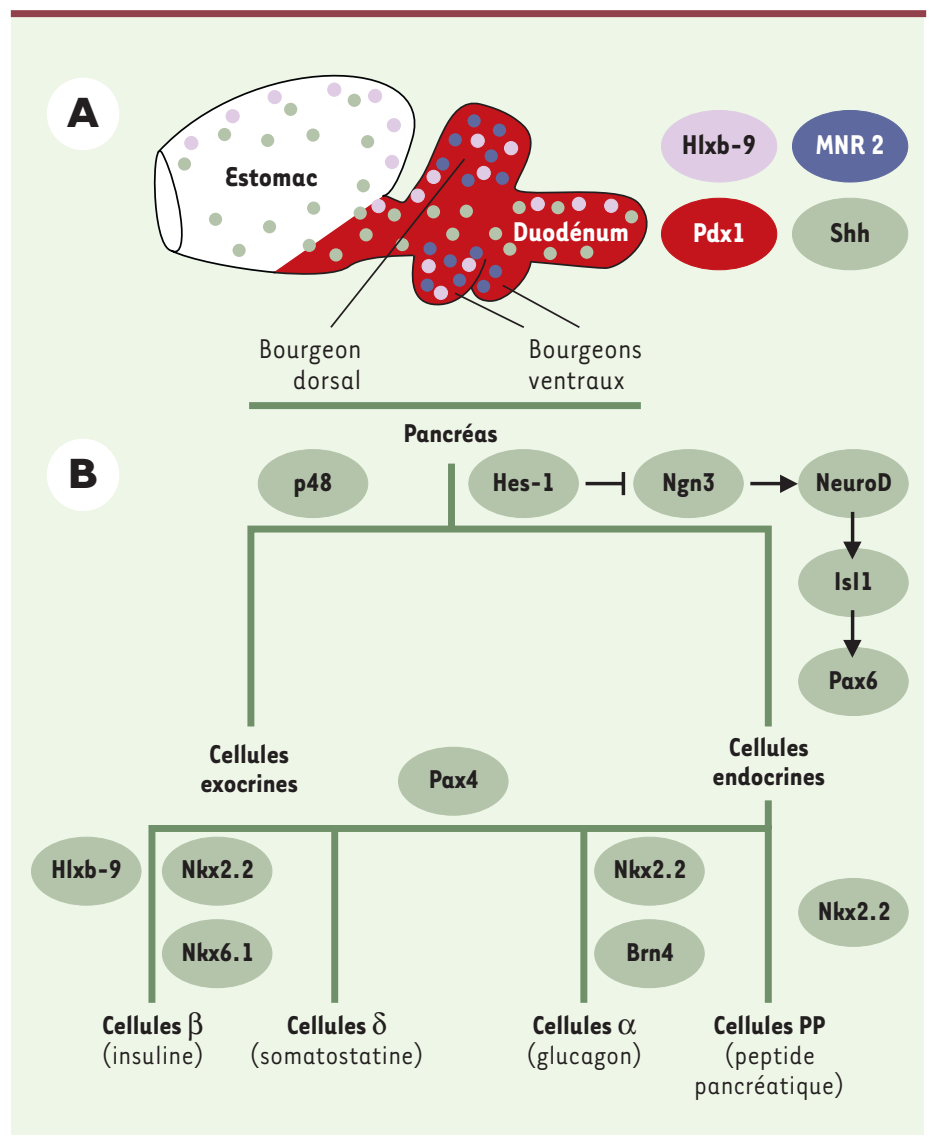

Après ce premier choix dichotomique, on ne sait pas bien comment les sous-types de cellules endocrines se spécialisent. Chez l'homme et la souris, les cellules qui produisent l'insuline, le glucagon, la somatostatine et le peptide pancréatique représentent respectivement 75-80, $10-20,5$ et $1 \%$ des cellules endocrines du pancréas. L'observation de cellules co-exprimant plusieurs hormones suggérait un choix tardif des cellules endocrines [22]. Une des rares études de lignage a pourtant révélé récemment que les cellules $\alpha$ et $\beta$ adultes n'ont jamais co-exprimé l'insuline et le glucagon au cours de leur vie, ce qui suggère que les cellules double positives vues chez l'embryon ne contribuent pas à l'organe mûr [23]. Les cellules $\beta$ dérivent de progéniteurs ayant exprimé le peptide pancréatique alors que ce n'est pas le cas pour les cellules $\alpha$. On pense que, comme dans la crête neurale, les progéniteurs restreignent progressivement leurs potentialités. Le lignage $\alpha$ se sépare précocement, un

Figure 3. Différenciation des cellules endocrines et exocrines. $A$. Les bourgeons du pancréas, dorsaux et ventraux, se développent à la charnière entre estomac et duodénum, dans le domaine qui exprime $P d x 1$. La protéine Shh est exprimée dans la quasi-totalité de l'épithélium digestif à l'exception du pancréas. Cette exclusion semble jouer un rôle important dans le développement du pancréas. Hlxb9 serait impliqué dans la formation des bourgeons pancréatiques. Chez le poulet, le gène MNR2, très similaire à HIxb9, est exprimé dans la totalité des bourgeons pancréatiques. B. Facteurs de transcription impliqués dans les différentes étapes de différenciation en cellules exocrines et endocrines. L'expression du facteur de transcription p48 est le premier signe de différenciation des cellules exocrines [16]. Certains gènes sont nécessaires à la différenciation de l'ensemble des cellules endocrines. Ainsi, le gène proneural ngn3, dont l'activation dépend de la voie de signalisation Notch/Delta, induit successivement un autre gène proneural Neuro $D$, puis les gènes paired homeodomain Pax6 et isl] $[13,20,24]$. Tous ces gènes sont induits par ngn3 et absents du pancréas lorsque ngn3 est inactivé [25]. D'autres gènes sont spécifiques de certains lignages. Ainsi, l'inactivation de Pax4 entraîne une augmentation du nombre de cellules $\alpha$ aux dépens des cellules $\beta$ et $\delta$ [24]. L'inactivation de Nkx2.2 empêche la différenciation des cellules $\beta$, réduit le nombre de cellules $\alpha$ et PP mais n'affecte pas le nombre de cellules $\delta$ [24]. En l'absence de ce gène, de nombreux précurseurs partiellement différenciés mais n'exprimant pas les hormones s'accumulent. Nkx6.1 et Hlxb9 affectent uniquement les cellules $\beta[10,24]$. Lorsque Nkx6.1 est inactivé, les quelques cellules $\beta$ qui apparaissent précocement se forment mais la majorité, qui se différencient vers $\varepsilon 13$ n'apparaissent pas. En l'absence de Hlxb9, il apparaît trois fois moins de cellules exprimant l'insuline que dans les embryons normaux [10]. En l'absence de Pdxl, aucune cellule à insuline n'est observée alors que des cellules à glucagon se différencient [6]. 
phénomène qui semble faire intervenir le gène Pax4 [24]. Un inventaire des différents types de progéniteurs et de leurs capacités de prolifération fait actuellement défaut.

\section{Une cascade de facteurs de transcription}

Un rapide examen des facteurs de transcription exprimés dans le pancréas révèle d'étonnantes similitudes avec le tube neural. On a d'ailleurs cru pendant longtemps que les cellules endocrines du pancréas dérivaient de la crête neurale et non de l'endoderme. Les facteurs de transcription à homéodomaine Pax6, Nkx2.2, Nkx6.1, Isl1, Hlxb9 et MNR2, et de type basique hélice-tour-hélice Ngn3 et NeuroD, sont exprimés dans les deux organes. Leur expression dans le tube neural, leur fonction dans la différenciation des soustypes de neurones et, dans une certaine mesure, leurs relations épistatiques ont été bien caractérisées. Dans le pancréas, les cellules d'un type particulier ne se différencient pas toutes en même temps. Le pancréas est donc composé pendant longtemps d'un mélange de cellules à des stades de développement très hétérogènes. II est par conséquent difficile de savoir quel gène est caractéristique d'une étape ou d'une voie de différenciation par la simple étude de son expression. Une première étape a été de déterminer si ces gènes sont exprimés dans des progéniteurs capables de proliférer. Leur inactivation et leur expression ectopique nous renseignent sur ce pour quoi ils sont respectivement indispensables et suffisants et permettent de déterminer leurs relations épistatiques. La Figure 3 montre que certains facteurs de transcription sont nécessaires à la différenciation de l'ensemble des cellules endocrines alors que d'autres sont spécifiques de certains lignages, et résume ce qui est connu de leur fonction.

De nombreux facteurs de transcription nécessaires à l'organogenèse du pancréas, tels que $\mathrm{Pdxl}, \mathrm{p} 48$, isll, Pax6, persistent chez l'adulte et activent la transcription des hormones et des enzymes [24]. Des mutations hétérozygotes dans certains de ces gènes ont été identifiées dans certains types de diabète de type II dans lesquels la sécrétion d'insuline est réduite ( $P d x 1$ : MODY4, NeuroD) [24].

\section{Formation des îlots de Langerhans}

Les cellules endocrines se forment dans la partie distale des bourgeons épithéliaux ramifiés du pancréas et quittent l'épithélium à $\varepsilon 16,5$ (Figure 4). Elles migrent dans le mésenchyme et s'agrègent pour former les îlots de Langerhans. Des expériences de chimères d'agrégation chez la souris ont montré que les îlots sont d'origine polyclonale [27]. Leur architecture, conservée, semble être importante pour l'homéostasie du glucose [8]. Les cellules à insuline sont au centre des îlots alors que les cellules à glucagon, somatostatine et PP, sont à la périphérie (Figure 4).

La formation des îlots est dépendante des molécules d'adhérence et de la capacité des cellules à digérer la matrice extracellulaire. Cette dernière propriété est conférée par les métalloprotéases dont au moins deux sont exprimées dans le pancréas, MMP-2 et 9 [8]. Leur inhibition perturbe la formation des îlots [8]. Les MMP sont induites par l'EGF et peut-être par des membres de la famille de TGF $\beta$ [8]. Les îlots des souris dont la voie de signalisation de l'EGF est invalidée restent à proximité des canaux. Les intégrines $\alpha v \beta 3$ et $\alpha v \beta 5$, deux protéines transmembranaires qui permettent l'adhérence des cellules à la matrice extracellulaire, permettent la migration des cellules endocrines [28]. Les interactions entre cellules endocrines jouent aussi un rôle dans l'organisation des îlots. Ces interactions sont contrôlées par deux grandes familles de protéines : celle

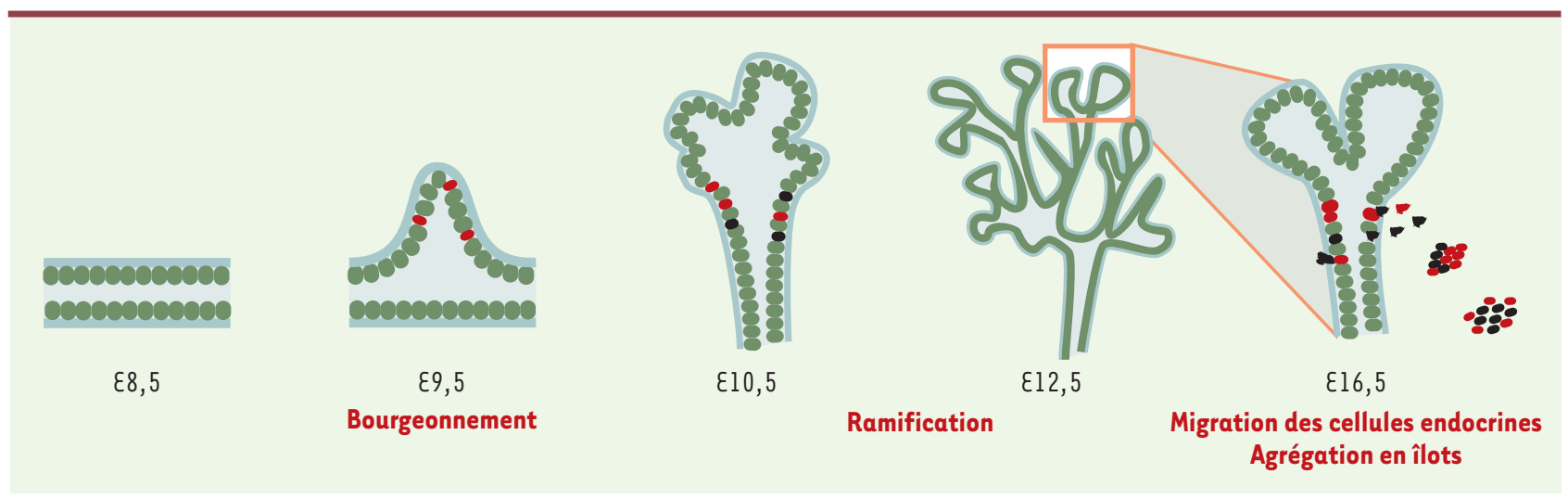

Figure 4. Morphogenèse du pancréas. Une coupe longitudinale du tube digestif montre à $\varepsilon 8,5$ les cellules de l'épithélium dorsal et ventral, en vert, et la lame basale en bleu. A 89,5 le bourgeon pancréatique dorsal est visible. Les cellules à glucagon, en rouge, ont commencé à se différencier. Le pancréas continue à se ramifier et les cellules à insuline, en noir, se différencient. A $\varepsilon 16,5$, les cellules endocrines quittent l'épithélium, franchissent la lame basale et s'agrègent en îlots dans lesquels les cellules $\beta$ sont au centre. 
des cadhérines qui relaye les interactions dépendantes du calcium et celle des CAM (cell adhesion molecules) qui en est indépendante [29]. Les cadhérines $\varepsilon$-, N- et $\mathrm{R}$ - sont exprimées dans les îlots et une forme dominante négative de la $\varepsilon$-cadhérine empêche l'agrégation des cellules endocrines [8]. En l'absence de N-CAM, les cellules $\alpha$ perdent leur localisation périphérique et se mélangent aux cellules $\beta[30,8]$. Les mécanismes intracellulaires qui coordonnent l'expression des molécules d'adhérence et, par conséquent, la migration sont méconnus. L'expression ectopique de la neurogénine 3 dans l'intestin grêle promeut le bourgeonnement dans le mésoderme et l'agrégation en îlots de cellules endocrines et il est possible que la différenciation endocrine et la formation des îlots soient coordonnées [13].

\section{Conclusions}

Que signifient ces données embryologiques, notamment dans le cadre du diabète ? Elles ont pour l'instant affecté le diagnostic du diabète en permettant d'identifier des gènes responsables de certains diabètes de type II. En ce qui concerne le diabète de type I, une thérapie envisagée pour le futur consisterait à remplacer les cellules $\beta$. Les découvertes embryologiques servent de base au développement de trois stratégies de production en masse des cellules $\beta$ à partir de cellules souches : (1) la première consiste à établir des protocoles de sélection des cellules qui se différencient en cellules $\beta$; (2) la seconde à introduire les gènes requis pour le développement des cellules $\beta$; (3) et la dernière à induire leur différenciation en présence de protéines sécrétées. Une meilleure connaissance des étapes de différenciation et de morphogenèse permettra de mieux contrôler la différenciation. Par exemple, lorsque Lumelski et al. [4] appliquent un protocole empirique de différenciation in vitro des cellules souches embryonnaires en cellules $\beta$, il est remarquable de constater que le taux de facteur de transcription Pdxl est extrêmement bas, ce qui pourrait expliquer pourquoi l'insuline est produite à un niveau 50 fois inférieur à la normale.

Le fonctionnement d'un organe repose sur la présence de cellules différenciées mais aussi sur leur organisation, ce qui, dans le cas du pancréas endocrine, correspond à la formation des îlots endocrines et des connexions sanguines et nerveuses. Il est plus réaliste à l'heure actuelle d'envisager d'injecter des cellules différenciées que de greffer un organe neuf produit in vitro. La greffe d'îlots de Langerhans semble d'ailleurs plus efficace que celle de l'organe complet. Ces îlots, injectés dans la veine porte, ne s'installent probablement pas dans le pancréas, mais coloniseraient plutôt le foie. Dans l'hypothèse de la production de cellules de remplacement in vitro, il est possible qu'il faille produire des îlots plutôt que des cellules $\beta$ seules.

Les progrès à venir dépendront de l'accroissement de nos connaissances sur les cellules souches, la culture des cellules endocrines et le développement du pancréas. Dans ce dernier domaine, les approches systématiques d'identification des gènes devraient permettre d'identifier les maillons manquants, et les approches fonctionnelles de reconstituer les cascades moléculaires impliquées dans la différenciation et la morphogenèse du pancréas. $\diamond$

\section{SUMMARY}

Different steps in pancreas development: toward diabetic therapies?

The formation of the pancreas from the endoderm layer takes place in different steps including the specification of the precursor territory, the outgrowth of pancreas buds, the differentiation of exocrine and endocrine cells, and the migration of endocrine cells which leads to islet formation. These processes are controlled by mesoderm-endoderm interactions and specific induction of a set of transcription factors. Deciphering the cascade of genes involved in beta-cell induction will provide essential tools to engineer cells for diabetes therapy. $\diamond$

\section{RÉFÉRENCES}
1. Shapiro AM, Lakey IR, Ryan $\varepsilon A$, et al. Islet transplantation in seven patients with type 1 diabetes mellitus using a glucocorticoid-free immunosuppressive regimen. $N$ Engl J Med 2000 ; $343: 230-8$.
2. Anderson DJ, Gage FH, Weissman IL. Can stem cells
cross lineage boundaries? Nat Med 2001 ; 7 : 393-5.
3. Gu D, Sarvetnick N. Epithelial proliferation and islet neogenesis in IFN- transgenic mice. Development 1993 ; 118 : 33-46.
4. Lumelsky N, Blondel 0, Laeng $P$, et al. Differentiation of

embryonic stem cells to insulin-secreting structures similar to pancreatic islets. Science 2001 ; 292 : 1389-94.

5. Jonsson J, Carlsson L, Edlund $T$, et al. Insulinpromoter-factor $l$ is required for pancreas development in mice. Nature 1994 ; 371 : 606-9. 
6. Offield MF, Jetton $T L$, Labosky PA, et al. PDX-1 is required for pancreatic outgrowth and differentiation of the rostral duodenum. Development 1996 ; 122 : 983-95.

7. Apelqvist A, Ahlgren U, Edlund $\mathrm{H}$. Sonic hedgehog directs specialised mesoderm differentiation in the intestine and pancreas. Curr Biol 1997; $7: 801-4$.

8. Kim SK, Hebrok M. Intercellular signals regulating pancreas development and function. Genes Dev 2001 ; 15 : 11-27.

9. Lammert $\varepsilon$, Cleaver 0 , Melton D. Induction of pancreatic differentiation by signals from blood vessels. Science 2001 ; 294 : 564-7.

10. Deutsch G, Jung J, Zheng $M$, et al. A bipotential precursor population for pancreas and liver within the embryonic endoderm. Development 2001 ; 128 : 871-81.

11. Harrison KA, Thaler J, Pfaff $\mathrm{SL}$, et al. Pancreas dorsal lobe agenesis and abnormal islets of Langerhans in Hlxb9-deficient mice. Nat Genet $1999 ; 23: 71-5$.

12. Li H, Arber S, Jessell TM, et al. Selective agenesis of the dorsal pancreas in mice lacking homeobox gene Hlxb9. Nat Genet $1999 ; 23$ : 67-70.

13. Grapin-Botton A, Majithia $A R$, Melton DA. Key events of pancreas formation are triggered in gut endoderm by ectopic expression of pancreatic regulatory genes. Genes Dev 2001 ; 15 : 444-54.

14. Thiery JP, Delouvee A, Gallin $W$, et al. Ontogenic expression of cell adhesion molecules: L-CAM is found in epithelia derived from the three primary germ layers. Dev Biol 1984 ; 102 : 61-78

15. Sosa Pineda B, Wigle JT, Oliver G. Hepatocyte migration during liver development requires
Proxl. Nat Genet $2000 ; 25$

254-5.

16. Krapp A, Knofler $M$, Ledermann B, et al. The bHLH protein PTFl-p48 is essential for the formation of the exocrine and the correct spatial organization of the endocrine pancreas. Genes Dev 1998 ; 12 : 3752-63.

17. Miralles F, Czernichow $P$, Scharfman R. Follistatin regulates the relative proportions of endocrine versus exocrine tissue during pancreatic development. Development 1998 ; 125 : 1017-24.

18. Apelqvist A, Li H, Sommer L, et al. Notch signalling controls pancreatic cell differentiation. Nature 1999 ; 400 : 877-81.

19. Jensen J, Pedersen $\varepsilon \varepsilon$, Galante P, et al. Control of endodermal endocrine development by Hes-1. Nat Genet $2000 ; 24: 36-44$.

20. Schwitzgebel VM, Schee DW, Conners JR, et al. Expression of neurogenin3 reveals an islet cell precursor population in the pancreas. Development $2000 ; 127: 3533-42$.

21. Krantz ID, Colliton RP, Genin $A$, et al. Spectrum and frequency of Jagged l (JAGI) mutations in Alagille syndrome patients and thei families. Am J Hum Genet 1998; 62 : 1361-9.

22. Teitelman $G$, Alpert $S$, Polak $J M$, et al. Precursor cells of mouse endocrine pancreas coexpress insulin, glucagon and the neuronal proteins tyrosine hydroxylase and neuropeptide $y$, but not pancreatic polypeptide. Development 1993 ; 118 : 1031-9.

23. Herrera PL. Adult insulinand glucagon-producing cells differentiate from two independent cell lineages. Development 2000 ; 127 : 2317-22.

24. Edlund H. Transcribing pancreas. Diabetes 1998 47: 1817-23.

25. Gradwohl G, Dierich $A$, LeMeur M, et al. Neurogenin 3 is required for the development of the four endocrine cell lineages of the pancreas. Proc Natl Acad Sci USA 2000 ; 97 : 1607-24.

26. Sander M, Sussel L, Conners $J$, et al. Homeobox gene Nkx6. 1 lies downstream of $N k \times 2.2$ in the major pathway of $\beta$-cell formation in the pancreas. Development $2000 ; 127: 5533-40$.

27. Deltour L, Leduque P, Paldi $A$, et al. Polyclonal origin of pancreatic islets in aggregation chimeras. Development 1991 ; 112 : 1115-21.

28. Cirulli V, Beattie GM, Klier G, et al. Expression and function of $\alpha v \beta 3$ and $\alpha v \beta 5$ integrins in the developping pancreas: roles in the adhesion and migration of putative endocrine projenitor cells. J Cell Biol $2000 ; 150$ : 1445-50.

29. Rouiller DG, Cirulli V, Halban PA. Uvomorulin mediates calcium-dependent aggregation of islet cells whereas calciumindependent cell adhesion molecules distinguish between islet cell types. Dev Biol 1991; 48 : 233-42

30. Esni F, Taljedal IB, Perl AK, et al. Neural cell adhesion molecule (N-CAM) is required for cell type segregation and normal ultrastructure in pancreatic islets. J Cell Biol 1999 ; 144 : 325-37.

\section{TIRÉS À PART}

A. Grapin-Botton 